\title{
Single Tooth Intrusion simplified with New Removable Orthodontic Appliance: Report of Two Cases
}

\author{
${ }^{1}$ Anil Kumar Mittal, ${ }^{2}$ Ruchi Sharma, ${ }^{3}$ Pratibha Garg, ${ }^{4}$ Amit Sidana
}

\begin{abstract}
Intrusion of single anterior tooth done by fixed orthodontic appliance is a tedious procedure for orthodontist as well as for the patient which may be quite expensive and time consuming. The use of a new modified removable orthodontic appliance is illustrated in two case reports in this article which is especially beneficial for such cases. This removable appliance is comprised of a labial bow of 21 gauge stainless steel wire with soldered high labial bow of 19 gauge stainless steel wire which has a soldered hook permitting the use of elastic. Adams clasp and pinhead clasps are used for retention of the appliance. During intrusive force application, one component is for intrusion of tooth and other component is for labial movement. This appliance is fabricated in such a manner that the labial movement is prevented by labial component of wire and is converted into pure intrusive force as much as possible. Force levels used for tooth movement are kept within physiologic limits while using this appliance. Although, this appliance is advantageous over fixed orthodontics due its simpler and quicker chair-side procedure and low cost of treatment associated with patient comfort, patient co-operation is must for the success of this therapy.
\end{abstract}

Keywords: Intrusion, Single tooth, Removable, Orthodontic, Appliance.

How to cite this article: Mittal AK, Sharma R, Garg P, Sidana A. Single Tooth Intrusion simplified with New Removable Orthodontic Appliance: Report of Two Cases. World J Dent 2014;5(4):232-236.

\section{Source of support: Nil}

Conflict of interest: None

${ }^{1,3}$ Private Practice, ${ }^{2}$ Senior Lecturer, ${ }^{4}$ Postgraduate (3rd Year)

${ }^{1}$ Department of Orthodontics, Mittal Dental Care and Orthodontic Centre, Panipat, Haryana, India

2Department of Orthodontics and Dentofacial Orthopedics, NIMS Dental College, Jaipur, Rajasthan, India

${ }^{3}$ Department of Pedodontics, Mittal Dental Care and Orthodontic Centre, Panipat, Haryana, India

${ }^{4}$ Department of Orthodontics and Dentofacial Orthopedics Saraswati Dental College, Lucknow, Uttar Pradesh, India

Corresponding Author: Ruchi Sharma, Senior Lecturer Department of Orthodontics and Dentofacial Orthopedics, NIMS Dental College, Jaipur, Rajasthan, India, Phone: 01426513102 e-mail: dr.sharmaruchi@rediffmail.com

\section{INTRODUCTION}

Intrusion of single anterior tooth done by fixed orthodontic appliance is a tedious procedure for orthodontist as well as for the patient. It may be quite expensive and time consuming. We have devised a new modified removable orthodontic appliance which is especially beneficial for such cases. By using this appliance, the intrusion of a single anterior tooth can be done in a small period of time and we can save the money and time of the patient.

This removable appliance is comprised of a labial bow of 21 gauge stainless steel wire with soldered high labial bow of 19 gauge stainless steel wire which has a soldered hook permitting the use of an elastic. Adams clasp and pinhead clasps are used for retention of the appliance. A $1 / 4^{\prime \prime}$ thin blue colored light intraoral elastic was used and approximately 1- 11/2 oz (ounce) of force was determined by Dontrix gauge before placing it on the hook to button.

\section{CASE REPORTS}

\section{Case 1}

An 18-year-old female patient, who presented with class II div 2 malocclusion as shown in Figure 1A and B in frontal and occlusal intraoral views, was given a removable active appliance for correction of retroclined central incisors. The extrusion of upper right central incisor of about $2 \mathrm{~mm}$ was noted at the later stages of treatment. Patient was not willing to use fixed appliance. Alternatively, she was given the modified removable appliance for intrusion of upper right central incisor.

Appliance was fabricated and placed intraorally (Fig. 2A). A button was bonded on the tooth to be intruded. Elastic was placed between the hook soldered on high labial bow and the button (Fig. 2B). Patient was instructed to replace the elastic with new one everyday. Correction was achieved within 3 weeks. No labial flaring of incisor occurred during the intrusion because of labial bow. The effect of appliance is shown in Figures $3 \mathrm{~A}$ to $\mathrm{D}$ in frontal and in occlusal views respectively. Figures $4 \mathrm{~A}$ and $\mathrm{B}$ clearly show the comparison between occlusal views of pretreatment and post-treatment stages. 


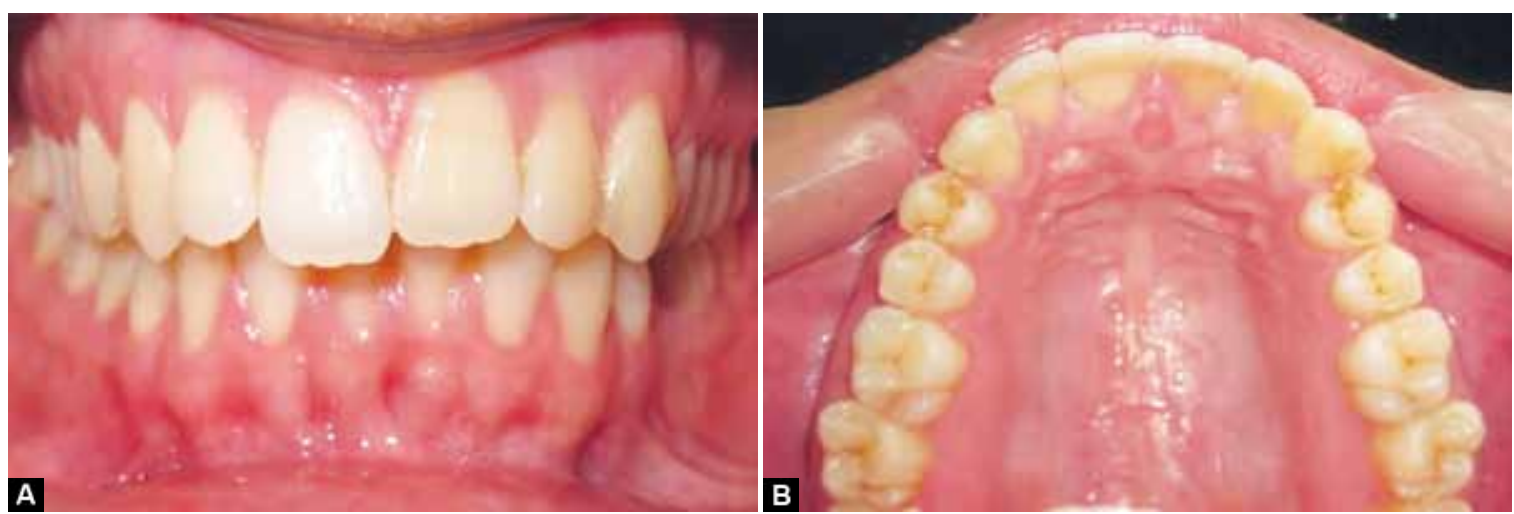

Figs 1A and B: Frontal and occlusal views before appliance placement
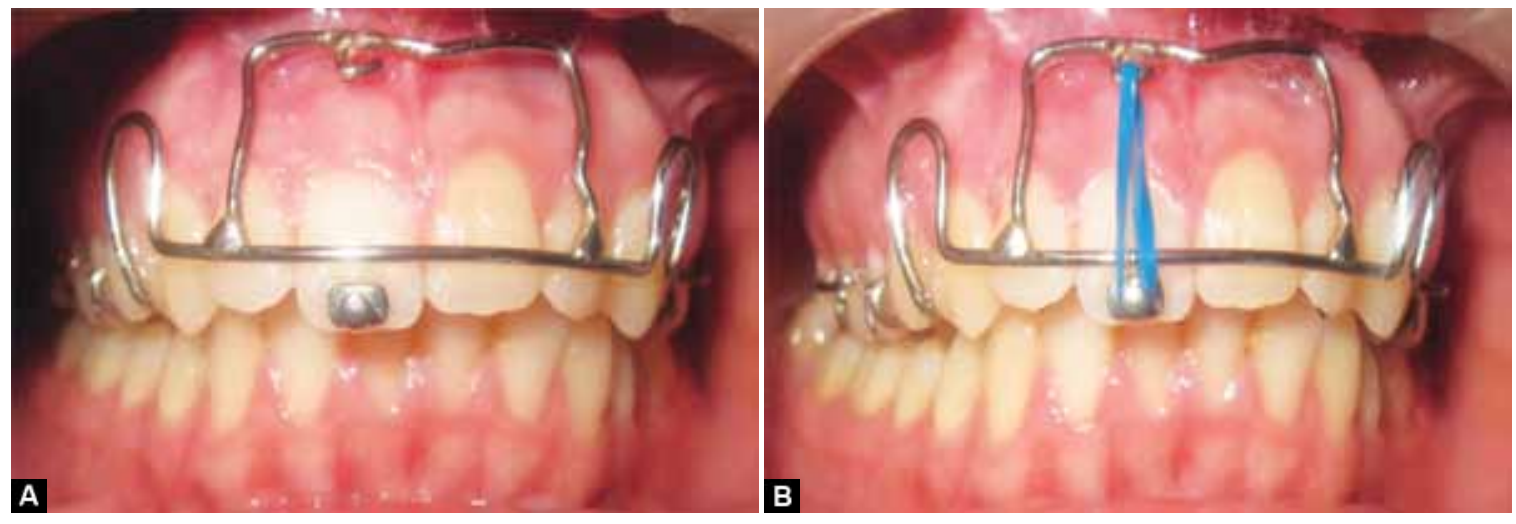

Figs 2A and B: Placement of the appliance and attachment of the elastic
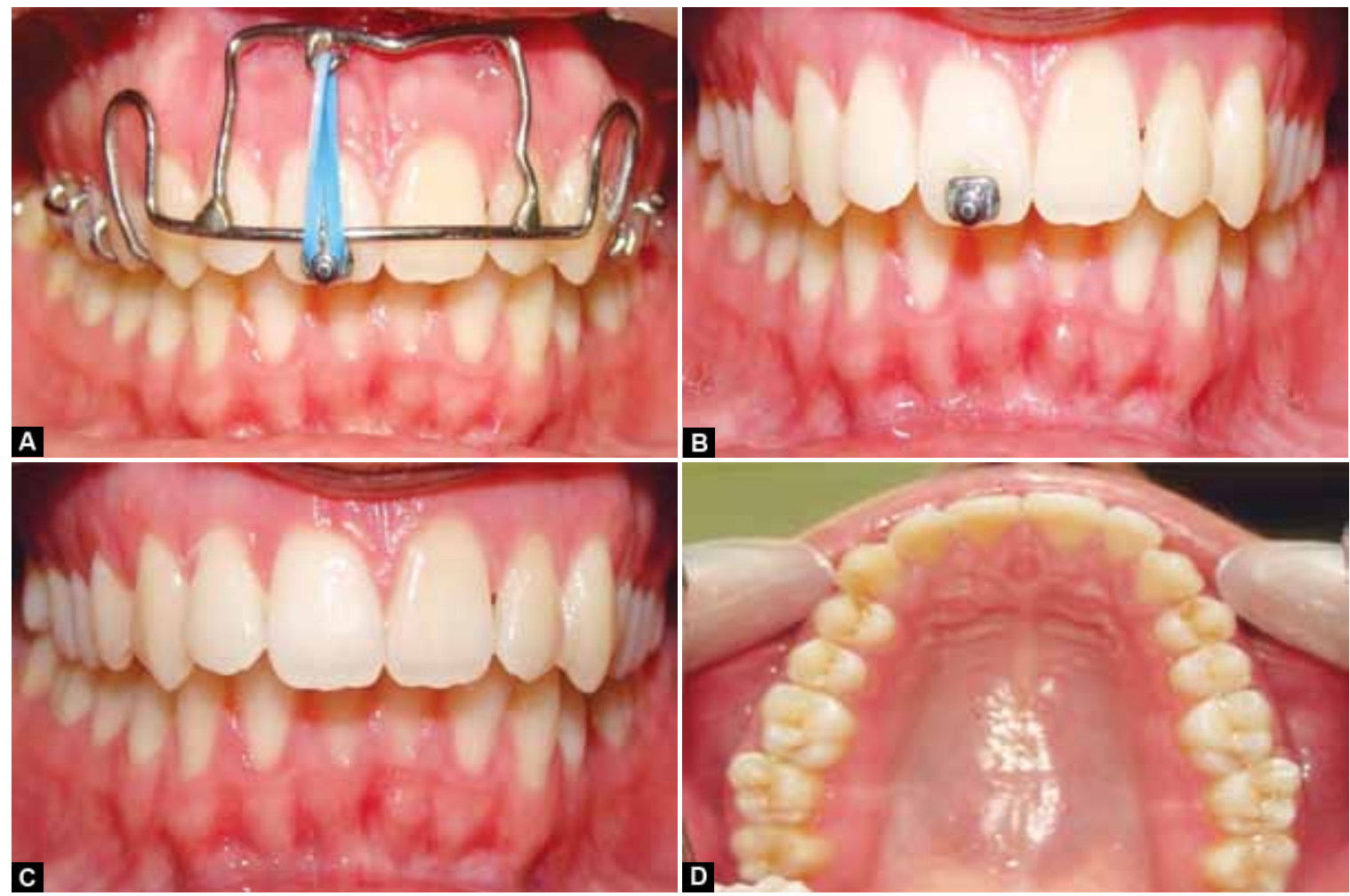

Figs $3 A$ to $D$ : (A to $C$ ) Frontal views after intrusion and (D) occlusal view after intrusion

\section{Case 2}

A 19-year-old female patient with extruded upper left central incisor of about $2.5 \mathrm{~mm}$ as shown in Figures $5 \mathrm{~A}$ and $\mathrm{B}$ in frontal and occlusal intraoral views was given the modified removable appliance for intrusion. Instructions were given how to wear the appliance and the elastic. Correction was achieved in about 21 days as shown in Figures 6A to C in frontal and (D) in 


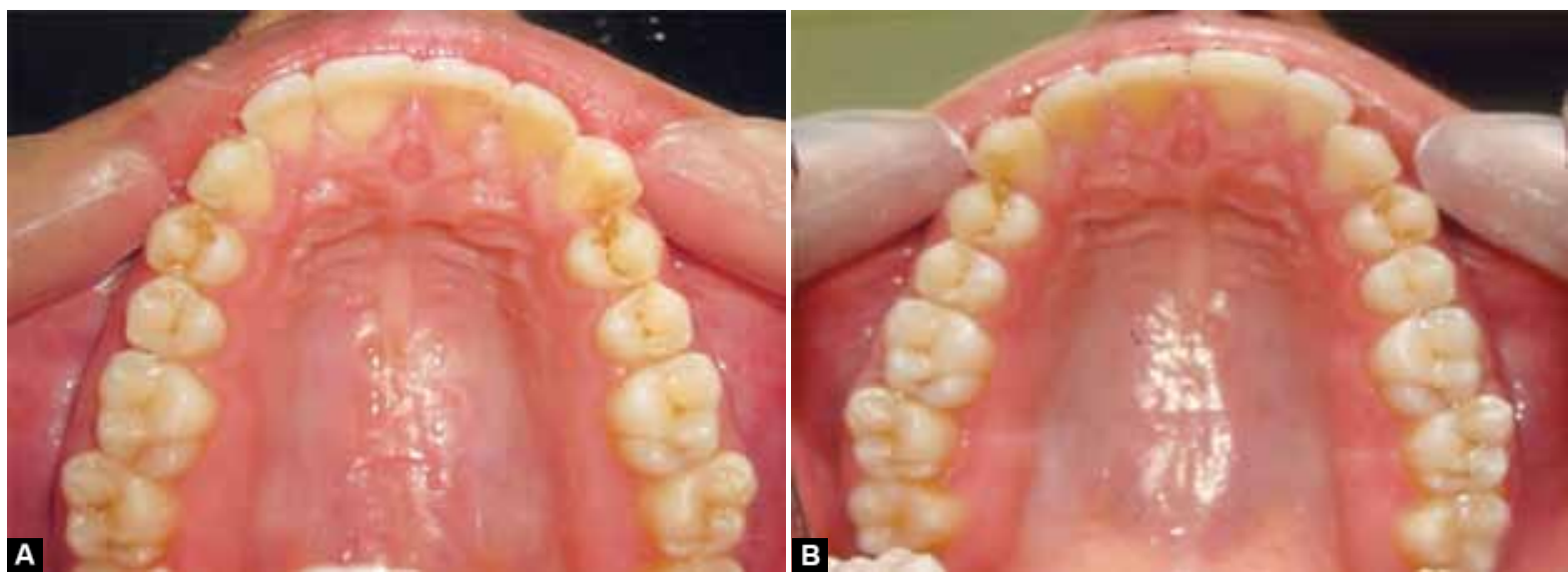

Figs 4A and B: Comparison of occlusal views before and after intrusion
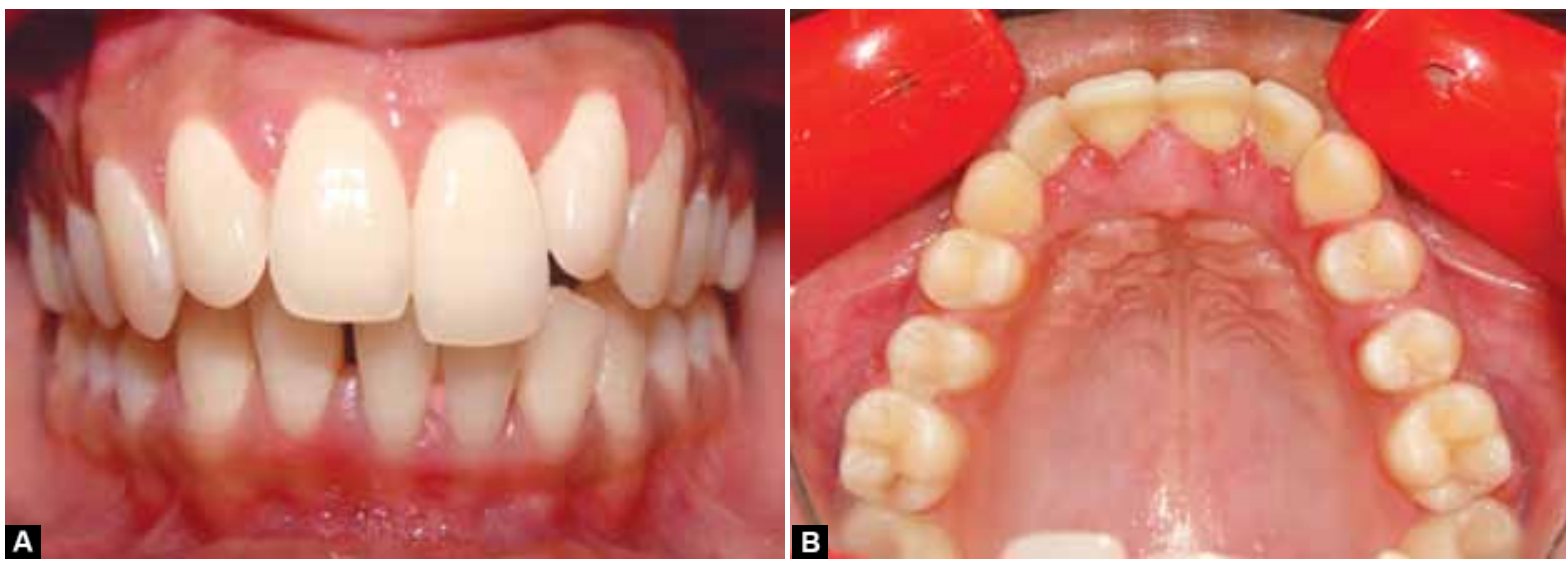

Figs 5A and B: Frontal and occlusal views before appliance placement
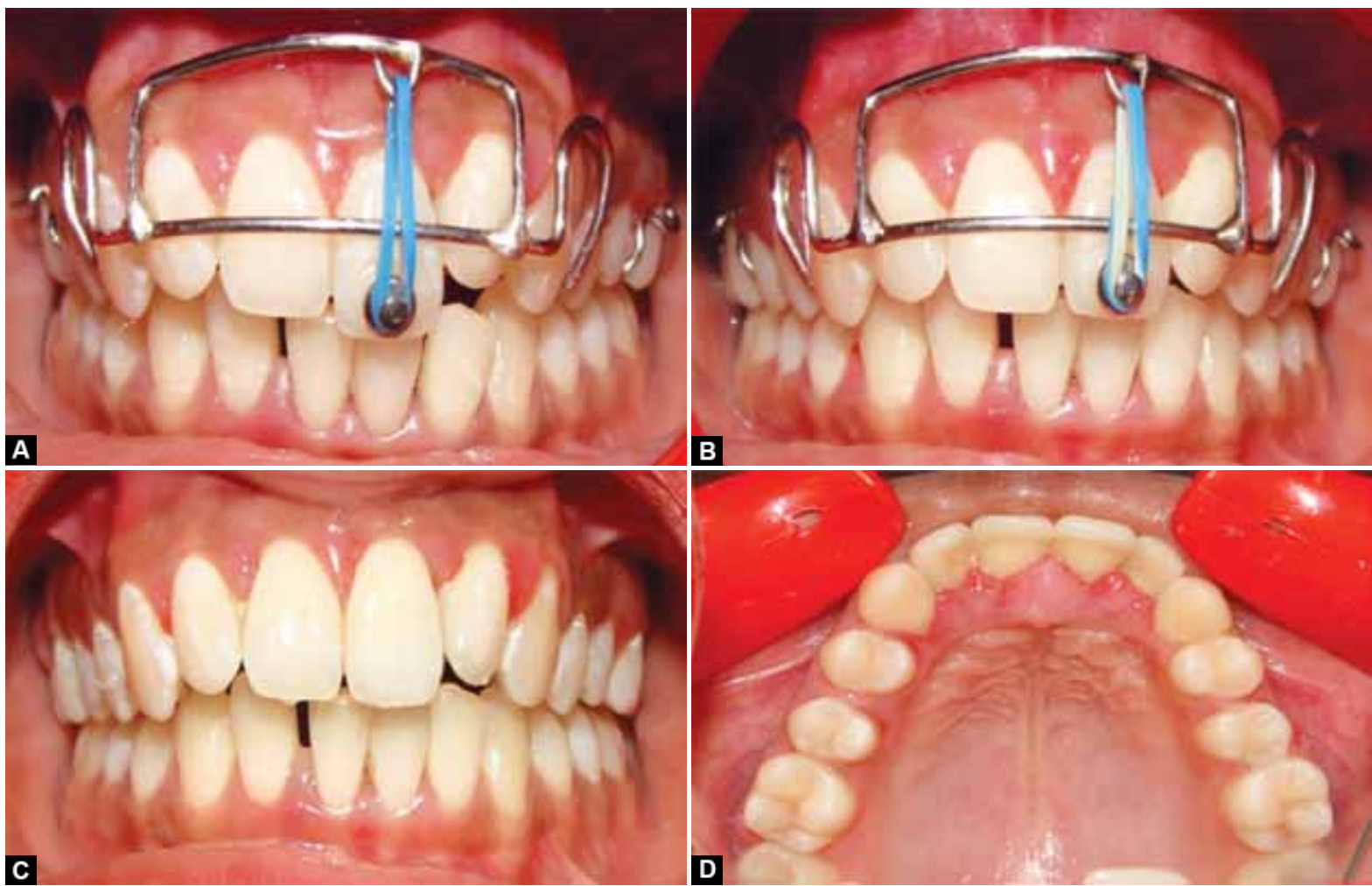

Figs $6 A$ to $D$ : (A to $C$ ) Frontal views before, during and after intrusion and (D) occlusal view after intrusion 

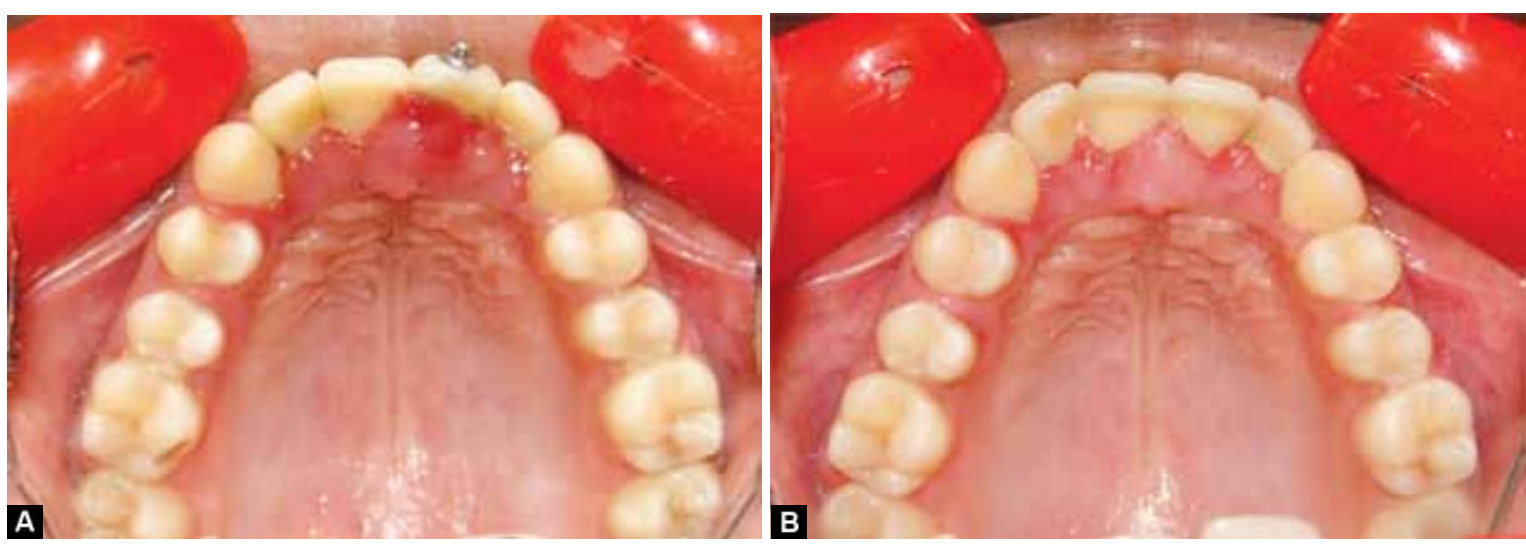

Figs 7A and B: Comparison of occlusal views before and after intrusion
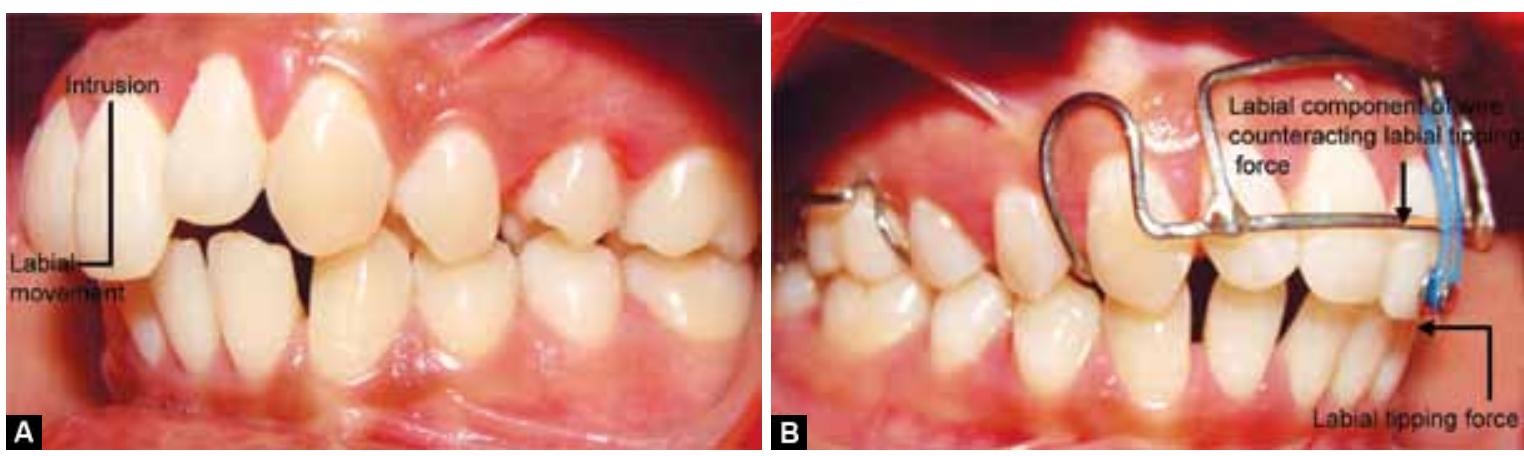

Figs 8A and B: (A) Components for intrusion and labial movement of tooth during intrusive force application and (B) labial tipping force counter acted by labial wire component of the appliance

occlusal views respectively. Figures 7A and B show the comparison between occlusal views of pre- and posttreatment stages.

\section{DISCUSSION}

It is a well known fact that during intrusive force application, one component is for intrusion of tooth which is vertical in direction and the other component is for labial movement (Fig. 8A). ${ }^{1}$ This other component for labial movement is produced by a crown-labial and root apex-lingual moment of force which can interfere with pure intrusion of tooth. To achieve maximum intrusion, the labial movement or flaring of tooth should be prevented and converted into bodily intrusion. This appliance is fabricated in such a manner that the labial moment of force is counter acted by labial component of wire and is converted into pure intrusive force as much as possible (Fig. $8 \mathrm{~B}$ ).

Force levels used for tooth movement are kept within physiologic limits while using this appliance. For many years it has been considered that it is almost impossible to produce pure orthodontic intrusion of teeth. It has become now clear that clinically we can achieve successful intrusion only when very light forces are applied to the teeth. ${ }^{2}$ The use of light force is recommended for intrusion so that appropriate pressure can be produced within the periodontal ligament because the force will be concentrated in a small area at the tooth apex. Only when the force is kept very light intrusion can be expected. ${ }^{2}$ A single anterior tooth will intrude by a force ranging from 20 to $30 \mathrm{gm}$. The light continuous force produces very short hyalinization periods and the tooth will be intruded quite rapidly with least discomfort and damage to the investing tissues.

The force produced by elastics on a tooth or a group of teeth depends on its magnitude. The overall stress produced depends on the site of application, its distribution through the periodontal ligament, morphology of root, alveolar process and condition of tooth health, age and most importantly the co-operation of the patient. Since, this appliance is a removable appliance, the important thing that should be taken care of is that the patient should be motivated and educated well to wear the appliance and elastic continuously except while brushing. We should completely ensure that the patient can place his or her elastic easily while changing it every day and that remains in place. If broken, the elastic should be changed immediately. It is also helpful to eat with the elastic in place. Sometimes, however, this is not possible. If the patient removes the elastic to eat, he or she should remember to replace that as soon as possible.

As far as the safety aspects of this appliance are concerned, no adverse effects, such as pain, irritation or 
tissue (either hard or soft) trauma were noted in patients after wearing this appliance. The framework of this appliance is constructed in such a way that it minimally interferes with speech and esthetics as compared to labial fixed orthodontic appliances. It is also noteworthy that allergic stomatitis from rubber bands is known to occur to those who are allergic to latex. ${ }^{3}$ History of allergies is useful in such situations and non-latex elastics should be used.

\section{CONCLUSION}

Although, this appliance is advantageous over fixed orthodontics due its simpler and quicker chair-side procedure and low cost of treatment associated with patient comfort, patient co-operation is must for the success of this therapy.

\section{REFERENCES}

1. Nanda R, Kuhlberg A. Management of deep overbite malocclusion. In: Nanda R, editor. Biomechanics and esthetic strategies in clinical orthodontics. Elsevier Saunders; 2005:141.

2. Proffit WR, Fields HW, Sarver DM. Contemporary orthodontics. 4th ed. Missouri: Mosby imprint, Elsevier; 2007: 340-341.

3. Kelso JM. Allergic contact stomatitis from orthodontic rubber bands. Ann Allergy Asthma Immunol 2007;98(1):99-100. 